\title{
¿SE PUEDEN EJERCER DE FORMA OMISIVA LAS REGLAS QUE CONFIEREN PODERES? *
}

\author{
Horacio José Alonso Vidal \\ Universidad de Alicante
}

RESUMEN. Este trabajo tiene el objetivo de demostrar cómo el poder jurídico puede ser ejercido no sólo de forma activa sino también mediante una omisión. Tomando como punto de partida la concepción de las reglas que confieren poderes de los profesores ATIENZA y RUIZ MANERO, así como un concepto de omisión tomado de vON WRIGHT, el autor argumenta, a través de varios ejemplos, cómo es posible ejecutar, mediante una omisión, reglas que confieren poderes de ejercicio facultativo. Finalmente, el trabajo analiza aquellos supuestos en los que el ordenamiento jurídico, ante determinadas conductas pasivas del agente a la ejecución de un poder de ejercicio obligatorio, establece una norma puramente constitutiva que atribuye un efecto jurídico a esa abstención, sin que ese caso pueda ser considerado como el ejercicio de una regla que confiere poder mediante una omisión. sión.

Palabras clave: Reglas que confieren poderes, normas puramente constitutivas, omi-

ABSTRACT. The purpose of this paper is to demonstrate how a legal power can be executed not only through an action but also by omission. Taking as starting point the conception about power conferring rules of ATIENZA and RUIZ MANERO and a conception of omission taken from VON WRIGHT, the author argues, using some samples, how is possible to execute a facultative power conferring rule by omission. Finally, the paper analyses those cases in which the legal system, before certain passive conducts of the agent concerning the execution of a power of obligatory exercise, establishes a purely constitutive norm that attributes a legal effect to that abstention, without being possible to consider that passive conduct an exercise of a power-conferring rule by omission.

Keywords: Power conferring rules, purely constitutive rules, omission.

\footnotetext{
* Fecha de recepción: 30 de noviembre de 2008. Fecha de aceptación: 18 de diciembre de 2008.
} 


\section{INTRODUCCIÓN}

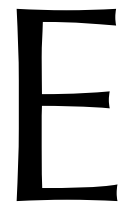

ste trabajo tiene el objetivo de mostrar cómo el poder jurídico puede ser ejercido no sólo de forma activa sino también mediante una omisión.

He estructurado la exposición en dos apartados: en el primero de ellos desen el segundo, —que constituye la parte original del estudio-, explicaré de qué manera las reglas que confieren poderes se pueden ejercer omisivamente.

En lo que a los fundamentos teóricos se refiere, partiré de la concepción de las reglas que confieren poderes como reglas anankástico-constitutivas ofrecida por ATIENZA y RUIZ MANERO, centrándome en el análisis estructural que estos autores realizan de este tipo de normas. A continuación, y siguiendo fundamentalmente a VON WRIGHT, expondré un concepto de omisión que me permitirá justificar la posibilidad de que las reglas que confieren poderes puedan ser ejercitadas omisivamente y no sólo de forma activa, pues la omisión, al igual que la acción, puede dar lugar a un resultado.

En la segunda parte del trabajo aplicaré los fundamentos teóricos antes citados para demostrar mi tesis sobre la modalidad omisiva de ejercicio de las reglas que confieren poderes. La estrategia que seguiré al respecto será la siguiente: en primer lugar, expondré diversos ejemplos de cómo es posible ejecutar mediante una omisión reglas que confieren poderes de ejercicio facultativo. Como ejemplos de esta posibilidad, estudiaré la institución de la tácita reconducción en los contratos de arrendamiento, así como la facultad de los Ayuntamientos de adoptar o no sus Planes Generales a la nueva normativa urbanística que se apruebe. A continuación, plantearé las dificultades para considerar que sea posible el ejercicio omisivo de las reglas que confieren poderes de ejercicio obligatorio.

Posteriormente abordaré una cuestión diferente de la anterior, pero directamente relacionada con lo expuesto. Dicho muy sintéticamente, se trata de cómo, en ocasiones, el ordenamiento jurídico, ante determinadas conductas pasivas de un agente de cara al uso de un poder de ejercicio obligatorio, establece una norma puramente constitutiva que atribuye un efecto jurídico a esa abstención, sin que exista en esta ocasión una regla que confiere poder ejecutada a través de una omisión. Ilustraré esta alternativa analizando las figuras del silencio administrativo y de la ficta confessio.

Por último, y a modo de recapitulación, expondré las conclusiones extraídas de mi desarrollo argumentativo.

\section{LA CONCEPCIÓN DE LAS REGLAS QUE CONFIEREN PODERES DE ATIENZA Y RUIZ MANERO}

ATIENZA y RuIZ MANERO consideran que las reglas que confieren poderes forman parte de una categoría más amplia, las normas constitutivas, que se contraponen a otra clase de normas, las regulativas ${ }^{1}$. En su opinión, las reglas que confieren poderes no son

${ }^{1}$ Véanse M. Atienza y J. Ruiz Manero, 2004: Las Piezas del Derecho, Barcelona: Ariel, 2. ${ }^{a}$ ed., pp. 70 y ss. 
normas deónticas o regulativas, y específicamente normas permisivas, tal como han sostenido autores como VON WRIGHT y ALCHOURRÓN y BULYGIN. La principal crítica que ATIENZA y RUIZ MANERO dirigen contra esta concepción es que no nos permite explicar los actos normativos irregulares (por ejemplo, una sentencia ilegal o una ley inconstitucional) ${ }^{2}$.

Como es sabido, ATIENZA y RUIZ MANERO rechazan igualmente las concepciones que pregonan que las reglas que confieren poderes sean disposiciones cualificatorias o reglas conceptuales o definiciones ${ }^{3}$, pues tales concepciones no serían lo suficientemente finas desde un punto de vista analítico ${ }^{4}$.

¿Qué son entonces las reglas que confieren poderes? Para responder a esta cuestión, ATIENZA y RUIZ MANERO parten del hecho de que toda norma jurídica obedece a un esquema condicional en el que puede distinguirse entre un antecedente y un consecuente. En las normas deónticas o regulativas el antecedente está formado por un estado de cosas en el que pueden estar presentes o no hechos institucionales Por su parte, el consecuente estaría integrado por una acción o un estado de cosas, natural o institucional, y un operador deóntico que modaliza esa acción o estado de cosas. Su forma canónica sería la siguiente:

«Si se da el estado de cosas $X$, entonces es obligatorio, prohibido, permitido la acción $Y$ para $Z$ ».

Sin embargo, acabo de señalar que ATIENZA y RUIZ MANERO rechazan categóricamente que las reglas que confieren poderes puedan reducirse a enunciados deón-

2 ATIENZA y RUIZ MANERO nos explican por qué: si consideramos a las reglas que confieren poderes como normas permisivas, entonces sólo nos quedan dos alternativas para dar cuenta de la existencia de actos normativos irregulares. La primera de ellas consiste en considerar que a los órganos jurídicos no les está permitido realizar actos normativos irregulares. La segunda de ellas supone lo contrario, esto es, que sí les estaría permitida la realización de tales actos. Optando por una u otra alternativa, la reducción de las reglas que confieren poderes a normas permisivas nos conduce a un dilema. En el primer caso, esto es, si optamos por considerar que no está permitida la realización de actos normativos irregulares, entonces no podremos explicar por qué tales actos normativos producen efectos jurídicos. Por su parte, ATIENZA y RUIZ MANERO analizan las consecuencias de optar por la segunda opción, esto es, de sostener que la realización de actos normativos irregulares está permitida, en relación con KELSEN, en su versión clásica de la Teoría Pura del Derecho, y su concepción de que las normas superiores determinantes de la producción normativa inferior permiten la edición de cualquier contenido. Así, glosando la opinión de KelSEN, ATIENZA y RuIZ MANERO escriben: «Todas las normas jurídicas, con excepción de las normas individuales (como las sentencias y también las resoluciones administrativas) que ordenan actos concretos de ejecución material, tendrían la forma de una disyunción entre su contenido expreso y una cláusula alternativa tácita que permitiría al órgano de producción de que se trate hacer caso omiso de tal contenido expreso».

${ }^{3}$ Básicamente las disposiciones cualificatorias o las reglas conceptuales implican la misma idea: permiten la identificación y el reconocimiento por el Derecho de las normas expresadas por las formulaciones normativas. Así, serían ejemplos de disposiciones cualificatorias o bien de reglas conceptuales o definiciones las normas relativas a la adquisición de la propiedad, que establecen cuándo puede entenderse que un sujeto $X$ ha adquirido la propiedad. Véanse M. AtienZA y J. Ruiz MANERo, Las Piezas..., op. cit., pp. 79-81.

${ }_{4}^{4}$ Estas posiciones, si bien presentan ventajas respecto a la reducción de las reglas que confieren poder a enunciados deónticos, incorporan en un mismo grupo disposiciones que presentan caracteres lo suficientemente diferenciados como para justificar su adscripción a dos categorías conceptuales diferentes. Así, sería preciso distinguir entre aquellas disposiciones cuya finalidad es aclarar cuándo estamos en presencia de una institución jurídica determinada, de aquellas otras cuya finalidad no se reduce a esa mera función de identificación, sino que se extiende a determinar cómo puede producirse (cumpliendo qué requisitos y realizando qué concreta acción) un ejemplo válido de esa institución. De ahí que para ATIENZA y RuIZ MANERO sea necesario distinguir al menos tres categorías diferentes: definiciones, reglas puramente constitutivas y reglas que confieren poderes. Véase ibídem, p. 81. 
ticos, y específicamente a normas permisivas. Así, el antecedente de las reglas que confieren poderes está formado por dos elementos: un estado de cosas que puede contener tanto hechos institucionales como naturales y una acción que puede igualmente ser natural o institucional. Su consecuente no consiste en una acción modalizada por un operador deóntico, sino en un hecho institucional al que ATIENZA y RUIZ MANERO denominan resultado institucional o cambio normativo. En contraposición con las normas regulativas, la forma canónica de una regla que confiere poder sería la siguiente:

«Si se da el estado de cosas $X$ y $Z$ realiza la acción $Y$, entonces se produce el resultado institucional o cambio normativo $R$ ».

Con posterioridad a las Piezas del Derecho, ATIENZA y Ruiz MANERo han añadido un cuarto elemento a su definición de las reglas que confieren poderes: el contenido. Esta adición fue efectuada por ATIENZA y RUIZ MANERO al percatarse que la anterior formulación canónica dejaba fuera el contenido proposicional expresado en el resultado normativo o cambio institucional. Pondré un ejemplo. En el supuesto que un juez ejerce la norma que le confiere el poder de dictar sentencia sobre una determinada materia, para que esa sentencia sea válida se requiere que su contenido se ajuste a Derecho y no simplemente su dictamen. Así, el esquema de la regla que confiere quedaría del siguiente modo 5 :

«Si se da el estado de cosas $X$ y el sujeto $Z$ realiza la acción $Y$ dando lugar al contenido $C$, entonces se produce el resultado institucional o cambio normativo $R$ ».

Analicemos a continuación cada uno de los elementos integrantes de la formulación canónica de las reglas que confieren poderes:

- Un estado de cosas X. El estado de cosas, tal como hemos expuesto anteriormente, puede estar integrado tanto por hechos naturales como institucionales.

- El agente $(Z)$ que ejercita la regla que confiere poder puede ser un sujeto particular o una autoridad pública ${ }^{6}$.

- Realización de la acción ${ }^{7}$. Y ATIENZA y Ruiz MANERO reconocen que hablar de la acción $Y$ es una simplificación, puesto que lo normal es que estemos «bien ante una conjunción de acciones (con un curso de acción o procedimiento), bien con una disyunción de cursos de acción, bien con alguna combinación de ambas» ${ }^{8}$. Pese a esta matización, es evidente que la propuesta de ATIENZA y RUIZ MANERO no contempla, al menos de forma explícita, que el resultado institucional o cambio normativo sea resul-

5 Véanse M. Atienza y J. Ruiz Manero, Seis..., op. cit., p. 724.

${ }^{6}$ Esta distinción, sin embargo, no es automáticamente asimilable a la que se puede efectuar entre ejercicio de poderes públicos frente a ejercicio de poderes privados o a la diferencia entre poder de heteronomía o poder de autonomía. En efecto, en ocasiones una autoridad puede ejercer un poder privado o autónomo $(v . g r$. una Administración Pública que realiza una compraventa con sujeción a las normas de Derecho privado). Y un sujeto particular puede ejercer un poder de heteronomía, como sucede con el ejercicio de la patria potestad. Véase, al respecto, M. Atienza y J. Ruiz Manero, Las Piezas..., op. cit., p. 98.

7 En cuanto al concepto de acción, lo analizaré más detenidamente en el apartado 3.1 de este trabajo.

8 M. Atienza y J. Ruiz Manero, Las Piezas..., op. cit., p. 106. Por otro lado, Atienza y Ruiz Manero, siguiendo una sugerencia formulada por D. GONZÁLEZ LAGIER, nos exponen que este elemento $Y$ también podría explicarse acudiendo al concepto de actividad, tal como éste está desarrollado en la obra de VON WRIGHT. Así, el curso de acción puede entenderse cómo la actividad que es necesario desplegar para realizar una acción, que en este caso sería el resultado institucional que se pretende alcanzar. Véase, ibídem, nota al pie de página. 
tado de una omisión. Los siguientes apartados están dedicados, precisamente, a intentar mostrar no sólo cómo es posible el ejercicio omisivo de reglas que confieren poderes, sino hasta qué punto algunas importantes instituciones de nuestro ordenamiento jurídico pueden reconstruirse adecuadamente contemplando expresamente esta posibilidad.

- El contenido. No me detendré en el comentario de este elemento, ya que la inclusión de este elemento en la formulación canónica de las reglas que confieren poderes por ATIENZA y RUIZ MANERO ya la he tratado anteriormente.

En resumen, las reglas que confieren poderes no son normas deónticas o regulativas, sino normas anankástico-constitutivas que pueden o deben ser utilizadas como reglas técnico-institucionales. Que las reglas que confieren poderes puedan o deban ser ejercitadas implica que su ejercicio puede estar modalizado, bien por una norma permisiva, y en tal caso, su ejercicio es facultativo, bien por una norma de mandato, y en ese supuesto, su ejercicio es obligatorio. De esta forma, la regla que confiere el poder a un juez para dictar sentencia sería de ejercicio obligatorio, mientras que la regla que confiere poder a un particular para otorgar testamento, sería de ejercicio facultativo. A fortiori, ATIENZA y RUIZ MANERO hacen especial hincapié en que la afirmación de que el ejercicio del poder esté modalizado por una norma regulativa no implica una contradicción con lo que se ha afirmado anteriormente, esto es, que las reglas que confieren poderes no son normas regulativas. ATIENZA y RUIZ MANERO reconocen que puede verse a las reglas que confieren poderes como una unidad funcional, en la que la presencia de una norma regulativa es ineludible, pero aun así las reglas que confieren poderes presentan por sí mismas caracteres propios que justifican su distinción de las normas regulativas. Así, mientras la función primaria de las normas regulativas es motivar o guiar la conducta de las personas, esa función sólo estaría presente de modo indirecto en las reglas que confieren poderes'. En primer lugar, porque mientras las normas regulativas establecen una guía determinada de la conducta, señalando, por ejemplo, cómo debemos comportarnos ante una situación, las normas que confieren poderes solamente nos indican cómo podemos o debemos comportarnos para conseguir un cambio normativo o un resultado institucional. En segundo lugar, porque las normas que confieren poderes serían, frente a las normas regulativas, doblemente condicionales, en el sentido que indican cómo debemos comportarnos antes determinadas circunstancias (al igual que sucede con las normas regulativas), pero a condición asimismo de que queramos o debamos conseguir un determinado resultado institucional o cambio normativo. De este modo, para ATIENZA y RUIZ MANERO, utilizando aquí como referencia la terminología kantiana, las normas regulativas de mandato actuarían en el razonamiento práctico como imperativos categóricos ${ }^{10}$, pues para quien acepta tales normas, ello es una razón suficiente para actuar conforme a esa norma. Las normas que confieren poderes, por el contrario, constituirían imperativos meramente hipotéticos: constituyen razones para actuar a condición de que el agente pretenda o deba alcanzar un resultado institucional o un cambio normativo, dadas ciertas circunstancias. A mayor abundamiento, si la norma que confiere poder es de ejercicio obligatorio para el agente (la razón para pretender alcanzar el resultado institucional o el cambio normativo es

\footnotetext{
9 Véanse M. Atienza y J. Ruiz Manero, Las Piezas..., op. cit., p. 90.

${ }_{10}$ Ibidem, p. 91.
} 
una norma de mandato) constituirá un imperativo hipotético-asertórico. Por su parte, si la norma que confiere poder es de ejercicio facultativo para el agente (la razón para pretender alcanzar el resultado institucional o el cambio normativo es un deseo o interés) constituirá un imperativo hipotético-problemático.

Hasta aquí me he centrado exclusivamente en la exposición de las reglas que confieren poderes. Sin embargo, ATIENZA y RUIZ MANERO distinguen dentro de la categoría de normas constitutivas otro de tipo de normas: las puramente constitutivas. Las normas puramente constitutivas se diferencian de las reglas que confieren poderes en que en su antecedente no figura una acción que haya de realizarse para producir el resultado. La simple presencia de determinadas circunstancias, un estado de cosas, da lugar al cambio normativo o resultado institucional. Su formulación canónica, en contraposición a las reglas que confieren poderes sería la siguiente:

$$
\begin{array}{ll}
\text { Dado el estado de cosas } \mathrm{X} \rightarrow \text { (surge) } & \begin{array}{l}
\text { el resultado institucional } \\
\text { o cambio normativo } R
\end{array}
\end{array}
$$

Por tanto, en las normas puramente constitutivas no se requiere para el surgimiento del resultado institucional o cambio normativo la ejecución por parte de un agente de una acción. Así sucede, por ejemplo, con la norma que establece que la personalidad civil se extingue por la muerte de las personas. Con independencia de que pueda haber estado implicada una acción en el fallecimiento (un suicidio, un asesinato), esa acción tendrá su propia valoración por el ordenamiento jurídico. Pero a los efectos de la extinción de la personalidad jurídica, el estado de cosas consistente en que alguien haya fallecido, y que normalmente no será el resultado de acción alguna, será el que dará lugar al cambio normativo ${ }^{11}$.

\section{LA OMISIÓN}

Antes de exponer cuál es el concepto de omisión que utilizaré en este trabajo conviene estudiar primero, aunque sea brevemente, el concepto de acción. No en vano, en la formulación canónica propuesta por ATIENZA y RUIZ MANERO de las reglas que confieren poderes la presencia de una acción es uno de sus elementos centrales como he señalado anteriormente.

\subsection{Concepto de acción}

Sin duda en la filosofía contemporánea la obra de VON WRIGHT es una de las que más atención ha prestado al estudio de la acción humana. El autor finés parte de la idea básica de que existe una relación entre algunas acciones humanas y algunos de los sucesos o cambios que se producen en el mundo, de tal suerte que muchos actos pueden describirse como la producción de un cambio a voluntad de un sujeto ${ }^{12}$.

\footnotetext{
${ }^{11}$ Ibidem, p. 87.

12 Sigo aquí fundamentalmente la exposición efectuada por D. GONZÁLEZ LAGIER, 1995: Acción y Norma en G. H. von Wright, Madrid: Centro de Estudios Constitucionales, pp. 59 y ss.
} 
Esta descripción, que incorpora la intención del agente de provocar un cambio como elemento nuclear, deja fuera otra clase de acciones, y específicamente las acciones no intencionales. Esta exclusión no es relevante para este trabajo, puesto que la propia estructura de las reglas que confieren poderes exige la intención en la ejecución de la acción. En efecto, las reglas que confieren poderes, como normas anankásticoconstitutivas, requieren que el agente realice una determinada acción a condición de que se pretenda alcanzar un determinado resultado institucional o cambio normativo, lo que siempre va a implicar la existencia de intencionalidad por parte del agente en su producción.

Dicho esto, los componentes definitorios de la acción (intencional) son, en una versión muy simplificada de la teoría de la acción de VON WRIGHT, los siguientes:

1. Un estado de cosas inicial, en el que se encuentra el mundo antes de que se efectúe la acción.

2. La intención o voluntad del agente de producir un cambio en ese estado de cosas inicial.

3. El proceso, consistente normalmente en una serie de movimientos corporales, dirigido a producir la alteración en el estado de cosas inicial.

4. Y por último, el resultado que se identificaría a estos efectos con el cambio producido en el mundo a consecuencia de la acción.

Estos cuatro componentes son los que permiten trazar una distinción importante para la teoría de la acción de VON WRIGHT: la diferencia entre el resultado y las consecuencias de una acción. Para el profesor de Helsingfors entre una acción y su resultado habría una relación conceptual, una conexión lógica, puesto que el propio concepto de acción se define como la producción de un cambio en el mundo a voluntad del agente; de modo que si tal cambio no se ha producido, entonces es que no se ha ejecutado la acción que daría lugar al mismo. Por su parte, otras transformaciones en el mundo posteriores al resultado, ya sean queridas o no por el agente, previsibles o no, son fruto, no ya de una conexión lógica sino de una relación causal, en lo que se conoce como consecuencias de la acción. Pondré un ejemplo. La muerte de un individuo a consecuencia de la acción de otro, es el resultado de dicha acción (la acción de matar), mientras la aflicción de su esposa (o su alegría) podría ser una de sus posibles consecuencias, amén de un reparto más o menos ordenado de su fortuna, si la hubiere.

Con lo expuesto hasta ahora se puede colegir el paralelismo existente entre la definición de acción efectuada por la teoría de VON WRIGHT y la concepción de las reglas que confieren poderes como normas anankástico-constitutivas sostenida por ATIENZA y RUIZ MANERO. En efecto, si el concepto de acción lo definimos básicamente en torno a la conexión lógica existente entre un estado de cosas inicial y un cambio en el mundo, la relación existente entre el cambio normativo o resultado institucional y el estado de cosas inicial en las reglas que confieren poderes también es de tipo conceptual. Ello implica que para que se produzca un determinado cambio normativo o resultado institucional se requiere la ejecución de una acción o curso de acción también determinados, de tal suerte que si el cambio normativo o el resultado institucional no se han producido, es que tampoco se ha ejecutado la acción o el curso de acción requerido por la regla que confiere poder para la producción del mismo. 


\subsection{Concepto de omisión}

Sin duda uno de los conceptos más complejos con los que se ha de enfrentar la Filosofía, no sólo del Derecho, es el de omisión. Las siguientes palabras de C. S. NiNO así lo atestiguan:

«La caracterización del concepto de omisión es sumamente obscura, como se advierte por el hecho de que estemos tentados tanto a ver a la omisión como una negación de la acción como a considerarla como una especie o modalidad de la acción» ${ }^{13}$.

No podemos profundizar aquí en todas las cuestiones que nos plantea el concepto de omisión, por ello me centraré solamente en dos problemas: la distinción entre omisión y «no hacer» y la aplicabilidad de los conceptos de resultado y consecuencias a la omisión ${ }^{14}$.

Una de las distinciones importantes en la obra de VON WRIGHT es la que traza entre la omisión y el simple «no hacer». Conforme a esta distinción, el agente está omitiendo una determinada acción cuando se abstiene de ejecutarla habiéndola podido llevar a cabo. La diferencia, por tanto, se establece en base al concepto de capacidad, esto es, sólo podemos omitir aquellas acciones que nos es posible ejecutar. La capacidad en este contexto no solamente se refiere a posibilidad fáctica, sino también a posibilidad jurídica, cuando del empleo de normas estamos hablando. Voy a mostrar esta idea de VON WRIGHT con un ejemplo:

Así, cuando un individuo nos dice que este verano ha decidido no pasar sus vacaciones en Alfa Centauri, no consideraremos que esté omitiendo la acción de viajar a la estrella más cercana al Sol y pasar allí sus vacaciones, ya que ese curso de acción está fuera de su alcance, al igual que para todos nosotros en el momento actual de la técnica aeroespacial. Simplemente, pensaremos que realmente no va a ir a Alfa Centauri. Sin embargo, si ese mismo individuo nos comenta que este verano no va a pasar sus vacaciones en Benidorm, como ha hecho los últimos años, sí que podremos afirmar que en este caso está omitiendo veranear en la población de la costa levantina.

En conclusión, para que podamos hablar de omisión de una acción y no de un simple no hacer, se exige que el agente tenga la capacidad fáctica y normativa de ejecutar la acción y sin embargo ésta no se lleve a cabo. Esta distinción entre capacidad fáctica y normativa me parece de suma importancia y volveré sobre ella al tratar las relaciones existentes entre las reglas que confieren poderes y las normas puramente constitutivas con la omisión.

Por otro lado, VON WRIGHT, en su obra Norma y Acción, afirma que de la omisión también puede predicarse un resultado y unas consecuencias ${ }^{15}$. El resultado de la omisión consistiría en que un cambio en el mundo no se ha producido al haberse el agente abstenido de ejecutar la acción que lo habría llevado a efecto, y en eso consistiría precisamente la intención del agente. También puede definirse como el estado de cosas

13 Ibidem, p. 75.

${ }^{14}$ Nuevamente insistimos en que estamos realizando una exposición muy sintética del pensamiento de VON WRIGHT sobre el particular. Para ver las vacilaciones y distintos problemas tratados por el autor finés respecto a la omisión con mayor profundidad puede consultarse ibidem, pp. 75 y ss.

15 Véase G. H. von Wright, 1970: Norma y Acción, Madrid: Tecnos. pp. 60 y ss. 
final, una vez ha tenido lugar la omisión. Por el contrario, las consecuencias de la omisión serían los efectos causales que se deriven del resultado producido por la omisión. Intentaré aclarar esta idea con otro ejemplo, cambiando el viaje a Alfa Centauri por la discusión de un matrimonio sobre si ir a la montaña o ir a la playa en vacaciones. Si el matrimonio finalmente acuerda, si es que eso es posible, que irán de vacaciones a la montaña, ello supondrá que están omitiendo la acción de veranear en la playa. El resultado de esa omisión será la ausencia de vacaciones en la playa, mientras que sus consecuencias pueden ser múltiples, como por ejemplo, el enfado del hijo adolescente de la pareja que ha visto cómo sus posibilidades lúdicas se reducían drásticamente al haber optado sus padres por ir de vacaciones a la montaña en lugar de ir a Benidorm.

En resumen, si la omisión puede dar lugar a un resultado y a unas consecuencias al igual que la acción, no existe ningún obstáculo conceptual para que el cambio institucional resultado del ejercicio de una regla que confiere poder sea efectuado a través de la omisión de una determinada acción ${ }^{16}$.

En la segunda parte de este trabajo voy a mostrar esta posibilidad a través de varios ejemplos, así como a analizar las relaciones entre las normas puramente constitutivas y las reglas que confieren poderes en referencia a la omisión.

\section{DIVERSOS EJEMPLOS DE EJERCICIO OMISIVO DE REGLAS QUE CONFIEREN PODERES}

Anteriormente he sostenido, siguiendo a VON WRIGHT, que acción y omisión comparten la producción de resultado, fruto de una conexión lógica o conceptual con la intención del agente, y unas consecuencias, fruto de los efectos causales del resultado. Es el momento de mostrar con diversos ejemplos cómo esa posibilidad teórica se da en la práctica. Para ello he tomado como referencia la clasificación de las reglas que confieren poderes que distingue entre aquéllas de ejercicio facultativo y aquéllas otras de ejercicio obligatorio.

\subsection{Omisión y reglas que confieren poderes de ejercicio facultativo}

Cuando examinaba el concepto de omisión he expuesto cómo la definición de omisión exigía un elemento adicional respecto a la acción: la capacidad del sujeto para realizar la acción omitida. Pues bien, en el ámbito de las reglas que confieren poderes de ejercicio facultativo nos podemos hacer una idea de cómo esa situación no va a ser infrecuente. Que el ejercicio de la regla que confiere poder sea facultativo supone que al agente le está permitido tanto realizar la acción que da lugar al resultado institucional o cambio normativo como su no ejecución, esto es, su omisión. Cuando el ordenamiento jurídico atribuye a esa omisión la producción de un resultado institucional o

${ }^{16}$ Esta posibilidad aparece apuntada por J. DELGADO ECHEVARRíA en su trabajo, «El concepto de validez de los actos jurídicos de Derecho Privado. Notas de teoría y dogmática», http://www.codigocivil.info/nulidad/lodel/document.php?id=79, p. 11, en el que sostiene que: «El poder puede ejercitarse también mediante una omisión, cuando tal es el acto previsto en la norma». 
un cambio normativo nos encontraremos ante una regla que confiere poder de ejercicio omisivo.

Así, Delgado ECHEVARRía ha puesto de manifiesto cómo nuestro ordenamiento tiene una gran tradición en otorgarle efectos a la omisión de una declaración de voluntad por el agente, tal como ocurre en los supuestos de tácita reconducción en los contratos de arrendamiento ${ }^{17}$. Veámoslo, utilizando la formulación canónica de las reglas que confieren poderes propuesta por ATIENZA y RUIZ MANERO:

Agente $Z$ (Arrendador o arrendatario)

Estado de cosas $X$ Acción- $Y$ Cambio normativo $R$ (vencimiento del contrato etc.) (envío requerimiento) (Resolución del contrato)

Agente $Z$ (Arrendador o arrendatario)

Estado de cosas $X$ .Omisión $Y$ Cambio normativo $R$

(vencimiento del contrato, etc.) (no se envía requerimiento) (Renovación del contrato)

En el primer supuesto, el arrendador o arrendatario llegado el vencimiento del arrendamiento, realiza una acción, el envío del requerimiento, para conseguir un resultado normativo: la resolución del contrato.

En el segundo supuesto, el arrendador o arrendatario llegado el vencimiento del arrendamiento, en lugar de enviar el requerimiento, algo para lo que están capacitados fáctica y normativamente en el sentido expuesto, no lo hacen, dando lugar a un resultado normativo diferente: la renovación del contrato.

Otro ejemplo de ejercicio omisivo de las reglas que confieren poderes de ejercicio facultativo estaría constituido por una clase de disposiciones muy comunes en nuestro ordenamiento urbanístico. Se trata de aquellas disposiciones que establecen que no es obligatorio para los municipios adaptar sus Planes Generales a la nueva normativa urbanística, una vez ésta ha sido aprobada ${ }^{18}$.

Agente $Z$ (Administración)

Estado de cosas $X$ Acción $Y$ Cambio normativo $R$

(nueva normativa) (adaptación del Plan General) (Plan General adaptado)

Agente $Z$ (Administración)

Estado de cosas $X$ Omisión- $Y$ Cambio normativo $R$

(nueva normativa) (Falta adaptación al Plan General) (Plan General no adaptado)

En el primer supuesto, la ejecución de los trámites de adaptación a la nueva normativa lleva aparejado un cambio normativo: un nuevo Plan General adaptado y homologado a la nueva normativa urbanística.

En el segundo supuesto, la no ejecución de los trámites de adaptación a la nueva normativa urbanística tiene como resultado un cambio normativo distinto: el mantenimiento del antiguo Plan General.

${ }^{17}$ Art. 10.1 de la Ley de Arrendamientos Urbanos de 1994.

${ }_{18}$ Véase, por ejemplo, la disposición transitoria segunda de la hoy derogada Ley 6/1994, de 15 de noviembre, Reguladora de la Actividad Urbanística. 
Este segundo ejemplo plantea la duda de que en realidad no se ha producido cambio normativo alguno, pues como decíamos se mantiene el Plan General hasta entonces vigente. Sin embargo, esa ausencia de adaptación sí que tiene un resultado propio, ya que implica, por ejemplo, que no se pueda reclasificar suelo no urbanizable en suelo urbanizable hasta que no se proceda a la adaptación, si quiera parcial, del Plan General a la nueva normativa urbanística. Y es perfectamente factible considerar que el equipo de gobierno de un Ayuntamiento busque voluntariamente no adaptar su Plan General a la nueva normativa urbanística, evitando de este modo, por ejemplo, la posibilidad de que se reclasifique suelo no urbanizable en su municipio. No obstante lo anterior, he de admitir que en los tiempos que corren este ejemplo pueda parecer claramente contrafáctico y por tanto un mal ejemplo.

\subsection{Omisión y reglas que confieren poderes de ejercicio obligatorio}

La posibilidad de que puedan ser ejercidas de modo omisivo las reglas que confieren poderes de ejercicio obligatorio presenta más dificultades. En efecto, siendo obligatorio para el agente alcanzar un determinado resultado institucional o cambio normativo dado cierto estado de cosas, el agente no está capacitado para dejar de realizar la acción que produce el resultado institucional o cambio normativo de que se trate. De este modo, la prohibición de non liquet, instituida por una norma de mandato, exige al juez resolver cada uno de los casos que se le presenten. El dejar de resolver un asunto supondría la violación de esa norma regulativa que modaliza el ejercicio del poder como obligatorio.

\section{LAS RELACIONES ENTRE LAS NORMAS PURAMENTE CONSTITUTIVAS Y LAS REGLAS QUE CONFIEREN PODERES Y LA OMISIÓN: EL CASO DEL SILENCIO ADMINISTRATIVO POSITIVO Y LA FICTA CONFESSIO}

En el apartado anterior he señalado cómo es posible que las normas que confieren poderes puedan ser empleadas por sus respectivos agentes por medio de una omisión. Me ocuparé ahora de cómo en ocasiones el ordenamiento jurídico, ante determinadas conductas pasivas de un agente de cara al uso de un poder de ejercicio obligatorio, establece una norma puramente constitutiva que atribuye un efecto jurídico a esa abstención, sin que exista en esta ocasión una regla que confiere poder ejecutada a través de una omisión.

El primero de los ejemplos que voy a analizar en ese sentido es el del silencio administrativo. Posteriormente, me ocuparé del supuesto de la ficta confessio.

\subsection{Omisión y silencio administrativo}

La institución del silencio administrativo aparece regulada en nuestro ordenamiento jurídico por el art. 43 de la Ley 30/1992, de 26 de noviembre, de Régimen Jurídico 
de las Administraciones Públicas y Procedimiento Administrativo Común, siendo una de las que con más frecuencia debe servirse el ciudadano en su trato con la Administración. Siguiendo al profesor MARTín MATEO ${ }^{19}$, el silencio positivo se explica por la inactividad de la Administración en el ejercicio de sus competencias y funciones. Así, para evitar mayores perjuicios al ciudadano nuestro ordenamiento jurídico atribuye a la falta de respuesta en un determinado plazo de una solicitud, un sentido, ya sea positivo o negativo. La significación positiva del silencio supone la estimación de la solicitud, existiendo un auténtico acto administrativo como si la declaración de la Administración hubiese sido expresa. La significación negativa, por el contrario, implica que el ciudadano debe entender desestimada la solicitud, a los efectos de que pueda interponer los recursos que procedan.

Ahora bien, la cuestión que se nos plantea es qué tipo de norma da cobertura a la institución del silencio administrativo. Hemos visto cómo la falta de respuesta por parte de la Administración produce un cambio institucional (la estimación de la solicitud en virtud del silencio) y un cambio en el status normativo del ciudadano (la desestimación de su petición, lo que le permite la interposición del correspondiente recurso). El cambio normativo o el resultado institucional derivan de una abstención, la falta de resolución expresa por parte de la Administración, lo que en principio parece indicar que estaríamos ante una regla que confiere poder que ha sido ejercida de modo omisivo. Expondré ahora cómo aparentemente la institución del silencio positivo encaja perfectamente con la formulación canónica de las reglas que confieren poderes ofrecida por ATIENZA y RUIZ MANERO:

Estado de cosas $X \rightarrow$ Agente $Z \rightarrow$ Omisión $Y \rightarrow$ Resultado o cambio institucional $R$

O lo que es lo mismo:

Presentación de una solicitud $+\rightarrow$ Administración $\rightarrow$ Falta de resolución expresa $\rightarrow$ Estimación de la solicitud

El problema que se plantea para considerar a la institución del silencio administrativo como el ejercicio omisivo de una regla que confiere poder es que, como he señalado anteriormente, la definición del concepto de acción u omisión requiere a su vez la presencia de intención, de voluntad por parte del agente en la producción del resultado. En ese sentido, el ejercicio de las potestades administrativas está regido por una norma de mandato que exige a la Administración que resuelva en todos los $\operatorname{casos}^{20}$, excluyendo por tanto la posibilidad de que normativamente un órgano administrativo o un funcionario pueda tener la intención de dejar de resolver un asunto, porque en todo caso la aplicación del silencio en un sentido u otro se hará de modo automático. O dicho de otro modo, puede que el funcionario o autoridad de que se trate no sea ejemplar y efectivamente tenga la intención de no resolver muchos asuntos, lo que sucede igualmente es que no le está permitido por el ordenamiento jurídico que se abstenga de

19 Véase R. Martín Mateo, 1999: Manual de Derecho Administrativo, Madrid: Trivium, pp. 330 y ss.

${ }^{20}$ Así lo establece el art. 42 la Ley 30/92, de 26 de noviembre, de Régimen Jurídico de las Administraciones Públicas y Procedimiento Administrativo Común. No obstante, esta afirmación debe matizarse en el sentido que en los casos en los que haya operado el silencio administrativo positivo, la Administración sólo puede resolver en el mismo sentido que el silencio, esto es, confirmándolo, de acuerdo a lo establecido en el art. 43.3 del mismo cuerpo legal. 
resolver. Por tanto, la conducta de la Administración cuando deja de resolver se trataría de un simple no hacer, ya que la conducta omisiva en estos supuestos no estaría permitida por el ordenamiento jurídico.

Por otro lado, nuestro ordenamiento, por razones institucionales y organizativas, ha regulado esa obligación de resolver de forma mucho menos categórica si la comparamos con la prohibición de non liquet que rige para los órganos judiciales. Los jueces y magistrados deben resolver todos los casos de su competencia que se les presenten. En caso contrario, estarían incurriendo en graves responsabilidades penales y disciplinarias. Por su parte, es una cuestión de hecho que los órganos administrativos y funcionarios dejan sin resolver en la práctica muchos de los asuntos que les son sometidos, confiando en que las reglas del silencio administrativo entrarán en juego y su falta de resolución, seguramente, no llevará aparejada sanción alguna ${ }^{21}$. Se produce aquí una suerte de contradicción pragmática entre lo que establece el ordenamiento jurídico que debe hacer la Administración: resolver en todos los supuestos, o lo que es lo mismo, que los poderes que le han sido conferidos deban ser ejercitados cada vez que se le plantee una solicitud, y los medios efectivamente articulados para garantizar esta obligación, que son mucho más endebles que los establecidos para asegurar que los jueces resuelvan todos los casos que se les presenten.

Ahora bien, si la aplicación del silencio administrativo no puede considerarse como una regla que confiere poder ejercida de modo omisivo: ¿De qué tipo de norma estamos hablando entonces? La respuesta a esta cuestión debemos buscarla en la idea inicial: el silencio administrativo es una institución pensada en beneficio del ciudadano, siendo su razón subyacente la pretensión de paliar los perjuicios irrogados por el incumplimiento por parte de la Administración de su obligación de resolver en plazo. De esta manera, el silencio administrativo funcionaría como una norma puramente constitutiva que no requiere la actuación (activa u omisiva) del agente (la Administración) para la producción del cambio normativo o resultado institucional y que permitiría al ciudadano considerar automáticamente que su solicitud ha sido estimada, o bien que ésta ha sido rechazada para poder interponer los recursos procedentes. Si el ejercicio obligatorio de los poderes conferidos a la Administración tuviese las mismas garantías que el que asegura el cumplimiento de la prohibición de non liquet de los jueces, no sería necesaria la vinculación de una regla puramente constitutiva a la falta de ejercicio por la Administración de una regla que confiere poder.

En consecuencia, el esquema de aplicación del silencio positivo sería el siguiente:

Si se da el Estado de cosas $X \rightarrow$ surge el $\rightarrow$ Resultado o cambio institucional $R$

O lo que es lo mismo:

Presentación de una solicitud + falta resolución expresa $\rightarrow$ estimación solicitud

${ }^{21}$ Si bien el art. 42.7 de la citada Ley 30/1992 establece que a los funcionarios o autoridades que incumplan la obligación de resolver en plazo les será exigible responsabilidad disciplinaria. 


\subsection{Omisión y ficta confessio}

Una situación similar a la del silencio positivo, aunque con ciertos matices, se produce respecto a la institución procesal de la ficta confessio ${ }^{22}$. La ficta confessio implica que en un proceso civil, demandante y demandado, deben comparecer al acto del juicio. En caso de no hacerlo, el tribunal podrá considerar reconocidos los hechos en que los que la parte que no comparezca hubiese intervenido personalmente y cuya fijación como ciertos le sea enteramente perjudicial. En este supuesto, la falta de comparecencia puede dar lugar a un efecto jurídico concreto: que se tenga a esa parte como confesa. Esa abstención, al igual que ocurría con el supuesto del silencio positivo, no puede entenderse como la aplicación omisiva de una regla que confiere poder de ejercicio obligatorio, pues la incomparecencia está prohibida por una norma de mandato, y de nuevo al agente no le está permitido omitir esa conducta, tratándose la incomparecencia al acto del juicio de un simple no hacer. La diferencia de esta institución frente a la del silencio administrativo, a la que antes he aludido, consiste que en el caso de la ficta confessio entra en juego una norma puramente constitutiva, cuyo resultado, el resultado institucional «incomparecencia», es a su vez el estado de cosas institucional que constituye la base para el ejercicio de una regla que confiere poder que faculta al juez para tener a la parte incompareciente por confesa o no. Acudiré una vez más a su formulación canónica para exponer el ejemplo:

Regla puramente constitutiva:

Si se da el Estado de cosas $X \rightarrow$ surge el $\rightarrow$ Resultado o cambio institucional $R$

O lo que es lo mismo:

Una de las partes citadas en un proceso civil $\rightarrow$ se considera que la parte ha no comparece al acto del juicio incomparecido.

Y como decía, la incomparecencia constituye a su vez el estado de cosas institucional que sirve de base a la eventual aplicación de una regla que confiere poder cuyo resultado podrá ser que se tenga a la parte por confesa o no.

Regla que confiere poder:

Agente $Z$ (Juez)

Estado de cosas $X$ Acción $Y$ Cambio normativo $R$

(Incomparencia de una de las partes) (valoración de la (Sentencia que tiene incomparencia) a la parte por confesa)

Agente $Z$ (Juez)

Estado de cosas $X$ Omisión- $Y$ Cambio normativo $R$ (Incomparencia de una de las partes) (no valoración de la incomparencia)

(Sentencia que no tiene a la parte por confesa)

${ }^{22}$ Véase el art. 304 de la Ley 1/2000, de 7 de enero, de Enjuiciamiento Civil. 


\section{CONCLUSIONES}

5.1. La concepción de las reglas que confieren poderes de ATIENZA y RUIZ MANERO parte del rechazo de las posiciones que las consideran normas regulativas, así como de aquellas otras que sostienen que este tipo de normas son definiciones, reglas conceptuales o disposiciones cualificatorias. En el primer caso, porque estas posturas son incapaces de explicar los actos normativos irregulares; y en el segundo, porque no permiten dar cuenta de las diferencias existentes entre las reglas que confieren poder y las meras definiciones. Como alternativa, ATIENZA y RUIZ MANERO consideran a las reglas que confieren poderes como normas anankástico-constitutivas, con una utilidad técnico-institucional. Este tipo de normas nos indican cómo se pueden o deben alcanzar ciertos resultados institucionales o cambios normativos. Ahora bien, la formulación canónica ofrecida por ATIENZA y RUIZ MANERO de las reglas que confieren poderes no contempla expresamente que sea la omisión de una acción la que dé lugar al cambio normativo o resultado institucional. Sin embargo, acudiendo al concepto de omisión que nos proporciona la obra de VON WRIGHT, he sostenido cómo una omisión también puede ser intencional y al igual que la acción dar lugar a un resultado y unas consecuencias, siempre y cuando el agente esté capacitado fáctica y jurídicamente para realizar la acción omitida, y la propia omisión no sea contraria al ordenamiento jurídico. Por tanto, no existe ningún obstáculo conceptual para que una omisión forme parte de la estructura de una regla que confiere poder, y en concreto de su antecedente.

5.2. Posteriormente, a través de varios ejemplos, he mostrado cómo esta posibilidad teórica se produce en la práctica, no sólo de forma episódica o puntual, sino que importantes instituciones de nuestro ordenamiento jurídico pueden reconstruirse a partir de su consideración como reglas que confieren poderes de ejercicio omisivo. Esta modalidad omisiva de aplicación de las reglas que confieren poderes se predica sin objeciones respecto a las reglas que confieren poderes cuyo ejercicio es facultativo. En efecto, siendo facultativo el ejercicio del poder, siempre se podrá llevar a cabo o bien omitir la acción que dé lugar al resultado institucional o cambio normativo. Cuando el ordenamiento jurídico atribuye un resultado a esa conducta pasiva, estaremos ante el ejercicio omisivo de la regla que confiere el poder, como sucede con la institución de la tácita reconducción. En efecto, llegado el vencimiento del contrato de arrendamiento, el envío o no de un requerimiento por parte de arrendador o arrendatario, implica, respectivamente, dos resultados normativos distintos: la resolución del contrato o bien su renovación. Respecto a las reglas que confieren poderes de ejercicio obligatorio, su aplicación mediante una omisión presenta más dificultades, pues siendo preceptivo el ejercicio del poder, el agente no puede abstenerse de realizar la acción que dé lugar al resultado institucional o el cambio normativo.

5.3. Finalmente, he analizado las relaciones entre las reglas que confieren poderes y las normas puramente constitutivas y la omisión. He sostenido que el silencio administrativo puede verse como una norma puramente constitutiva cuya finalidad sería paliar los efectos negativos de la falta de resolución en plazo. Esa falta de ejercicio de la regla que confiere poder por parte de la Administración no puede verse como una omisión en el sentido expuesto en este trabajo, porque el ordenamiento jurídico prohíbe a la Administración esa abstención, por lo que faltaría el requisito de la capacidad normativa, esto es, que no exista una norma de mandato que prohíba esa conducta pa- 
siva, para poder considerar que esa falta de resolución es una omisión. Asimismo, he analizado la vinculación de una regla puramente constitutiva a la falta de ejercicio por parte de la Administración como una suerte de contradicción pragmática, en el sentido que el ordenamiento jurídico establece que la Administración debe resolver expresamente en todos los casos, pero sin embargo articula una norma puramente constitutiva (el silencio administrativo) para cubrir los efectos perjudiciales de la inactividad administrativa, dando por descontado que se va a incumplir sistemáticamente la obligación de resolver.

En el caso de la ficta confessio, he analizado la situación que se produce cuando una de las partes en un proceso civil no acude al acto del juicio como una regla puramente constitutiva, que da lugar a un resultado institucional, la incomparencia, que constituirá el supuesto de ejercicio de una regla que confiere el poder al juez para, valorando las circunstancias del caso, considerar que se tenga a la parte por confesa o no.

\section{BIBLIOGRAFÍA}

Atienza Rodríguez, M., y Ruiz Manero, J., 2000: Ilícitos atípicos, Madrid: Trotta.

- 2003: «Seis acotaciones sobre una teoría de la validez jurídica», DOXA. Cuadernos de Filosofía del Derecho, n. ${ }^{\circ} 26$, Alicante.

- 2004: Las Piezas del Derecho, Barcelona: Ariel, 2. ${ }^{a}$ ed.

Delgado EchevarRía, J.: «El concepto de validez de los actos jurídicos de Derecho Privado. Notas de teoría y dogmática», http://www.codigo-civil.info/nulidad/lodel/document.php? id $=79$.

GonZÁlez Lagier, D., 1995: Acción y Norma en G. H. von Wright, Madrid: Centro de Estudios Constitucionales.

Martín Mateo, R., 1999: Manual de Derecho Administrativo, Madrid: Trivium, pp. 330 y ss. Von Wright, G. H., 1970: Norma y Acción, Madrid: Tecnos. 